\title{
Basis of selection of first and second line highly active antiretroviral therapy for hiv/aids on genetic barrier to resistance: a literature review.
}

\author{
Christine Katusiime ${ }^{1}$, Ponsiano Ocama ${ }^{2}$, Andrew Kambugu ${ }^{1}$
}

1. Makerere University, College of Health Sciences, Infectious Diseases Institute, Kampala, Uganda

2. Makerere University, College of Health Sciences, Department of Medicine, Kampala, Uganda.

\begin{abstract}
:
The effectiveness of combination antiretroviral therapy (cART) continues to improve as treatment choices expand with the development of new antiretroviral agents and regimens. However, the successful long-term treatment of HIV/AIDS is under threat from the emergence of drug-resistant strains to multiple agents and entire drug classes.
\end{abstract}

Key words: genetic barrier, highly active antiretroviral therapy, HIV resistance

DOI: http://dx.doi.org/10.4314/ahs.v14i3.25

\section{Introduction}

Long-term management of HIV/AIDS is at risk of increasing drug resistance(1). Booster protease inhibitor (PI) and thymidine analogue-containing regimens have a high genetic barrier to resistance (25). Non-thymidine-analogue triple nucleoside/nucleotide reverse transcriptase inhibitor (NRTI/NtRTI) combination regimens and Non-nucleoside reverse transcriptase inhibitors on the contrary have a low genetic barrier to resistance (6-9).

Unfortunately, several low genetic barrier agents select for mutations that confer broad class resistance (10, 11). The selection of the K103N and Y181C mutations cause loss of activity to all currently available NNRTIs. Most NRTIs are rendered inactive by the selection of the K65R mutation (12). The M184V mutation in addition causes loss of activity to both lamivudine (3TC) and emicitrabine (FTC), but has been shown to confer viral resensitisation to zidovudine (ZDV), stavudine (d4T) and tenofovir (TDF) (12) and delay in thymidine analogue mutations (TAMs) emergence (5). L74V mutation selection causes decreased antiviral activity of abacavir (ABC), didanosine (ddI), and zalcitabine (ddC), and when the mutated virus is selecting for both $\mathrm{L} 74 \mathrm{~V}$ and M184V, only the thymidine analogues (ZDV and $\mathrm{d} 4 \mathrm{~T}$ ) and TDF retain susceptibility (12). In this paper, we review literature focusing on the genetic barrier to resistance as the basis of selection of first and second line antiretroviral therapy.

\section{Corresponding author: \\ Christine Katusiime \\ Makerere University, College of Health \\ Sciences, Infectious Diseases Institute, \\ Kampala, Uganda \\ Email: katutina@yahoo.com}

\section{Methods}

A search of the SCOPUS and MEDLINE databases for articles on commonly used HAART and genetic barrier was done. The keyword 'genetic barrier' was cross-referenced with the keywords 'HIV' and 'HIV resistance'. Secondary references were also reviewed. Results were restricted to articles published in English between 1980 and 2009. The year 1980 was selected as the beginning of the period of interest to ensure that we captured an adequate amount of published literature.

\section{Results}

Based on the search terms, the searches yielded 185 articles eligible for inclusion. After titles and abstracts were reviewed, only 22 articles were reviewed in full, of which 9 presented data sufficient for inclusion in the final analysis.

\section{Discussion}

With growing access to CART in low-resource settings, treatment options in terms of better and less toxic medications is also increasing. Table I shows different literature discussing genetic barriers of various commonly used cART with their corresponding virologic suppression rate. The decision to use low or high genetic antiretroviral regimens as initial cART in treatment naïve patients is not definitive and is largely governed by availability especially in resource-limited settings. Booster PI-based regimens generally have a high genetic barrier to resistance $(1,13,15,16,18-20)$ and are recommended for use but gastrointestinal adverse drug effects precludes their use as first line therapy and is mainly reserved for second line therapy21. Selection of low genetic barrier combinations on the contrary, 
may result into broad class resistance hence reducing treatment options10, 11. Genetic barrierinformation however, may provide guidance to health workers on the selection of optimal cART regimens to improve the durability of CART and therefore ensure treatment success. Combinations of both low and high genetic barrier ART drugs are recommended. These combinations of high and low genetic barrier ART may produce potent first-line therapy. The ramification of potent cART is effective virologic control which is not easily rendered inactive in cases of poor adherence.

\section{Conclusion}

Different regimens have different genetic barriers to resistance and the recommendation would be to use combinations of low and high genetic barrier drugs for instance ZDV in combination with low genetic barrier drugs as first-line therapy. Use of low genetic barrier regimens as first line therapy confers broad class resistance over more than one group of ARVs. Use of high genetic barrier regimens as first line therapy on the contrary, requires a number of mutations to be rendered inactive and therefore will allow clinicians to be able to provide both support and adherence counselling so as to ensure treatment success for subsequent regimens.

\section{References:}

1. Luber A. Genetic barriers to resistance and impact on clinical response. MedGenMed 2005; 7(3):69.

2. Hsu RK, Wainberg MA. Do new protease inhibitors offer improved sequencing options? Issues of PI resistance and sequencing. J Acquir Immune Defic Syndr 2004; 35 Suppl 1:S13-21.

3. Kempf D, King M, Bernstein B et al. Incidence of resistance in a double-blind study comparing lopina$\mathrm{vir} /$ ritonavir plus stavudine and lamivudine to nelfinavir plus stavudine and lamivudine. J Infect Dis 2004; 189(1):51-60.

4. Eron Jr J. Thetreatment of antiretroviral-naive subjects with the 3TC/zidovudine combination: a review of North American (NUCA 3001) and European (NUCB 3001) trials. AIDS 1996; 10 Suppl 5:S11-9.

5. Melby T, Tortell S, Thorborn D et al. Time to appearance of NRTI-associated mutations and response to subsequent therapy for patients on failing $\mathrm{ABC} / \mathrm{COM}$. 8th Conference on Retroviruses and Opportunistic Infections. 2001:448.

6. Farthing C, Khanlou H, Yeh V. Early virologic failure in a pilot study evaluating the efficacy of abacavir, lamivudine and tenofovir in the treatment naive HIV- infected patients. Antivir Ther. 2003; 8(1):43.

7. Gallant J, Gerondelis P, Wainberg M et al. Nucleoside and nucleotide analogue reverse transcriptase inhibitors: a clinical review of antiretroviral resistance. Antivir Ther 2003; 8(6):489-506.

8. Landman R, Peytavin G, Descamps D et al. Low genetic barrier to resistance is a possible cause of early virologic failures in once-daily regimen of abacavir, lamivudine, and tenofovir: the Tonus study. Conference on Retroviruses and Opportunistic Infections. 2004; 11:52.

9. Ruane P, Luber A, Akil B et al. Factors influencing selection of K65R mutation among patients receiving tenofovir (TDF) containing regimens. 2nd IAS Conference on HIV Pathogenesis and Treatment. Antivir Ther 2003; 8(1):582.

10. D'Aquila R, Schapiro J, Brun-Vezinet F et al. Drug resistance mutations in HIV-1. Top HIV Med. 2003; 11(3):92-96.

11. Hirsch M, Brun-Vezinet F, Clotet B et al. Antiretroviral drug resistance testing in adults infected with human immunodeficiency virus type 1: 2003 recommendations of an International AIDS Society-USA Panel. Clin Infect Dis 2003; 37(1):113-128.

12. Lanier R, Irlbeck D, Ross L et al. Prediction of NRTI options by linking reverse transcriptase genotypes to phenotypic breakpoints.10th Conference on Retroviruses and Opportunistic Infections. 2003: 586.

13. Di Giambenedetto S, Torti C, Prosperi M et al. Effectiveness of antiretroviral regimens containing abacavir with tenofovir in treatment-experienced patients: predictors of virological response and drug resistance evolution in a multi-cohort study. Infection 2009; 37(5):438-444.

14. Brenner G, Coutsinos D. The K65R mutation in HIV-1 reverse transcriptase: genetic barriers, resistance profile and clinical implications. HIV Ther 2009; 3(6):583-594.

15. Chandwani A, Shutter J. Lopinavir/ritonavir in the treatment of HIV-1 infection: A review. Ther Clin Risk Manag 2008; 4(5): 1023-1033.

16. Barragan P, Podzamczer D. Lopinavir/ritonavir protease inhibitor for HIV-1 treatment. Expert Opin Pharmacother 2008; 9(13): 2363-2375.

17. Sirivichayakul S, Phanuphak P, Pankam T, O-Charoen R, Sutherland D, Ruxrungtham K. HIV drug resistance transmission threshold survey in Bangkok, Thailand. Antivir Ther 2008; 13 Suppl 2: S109-113.

18. Martinez-Cajas JL, Wainberg MA. Proteaseinhibitor resistance in HIV-infected patients: Molecular and Clin- 
ical perspectives. Antiviral Res 2007; 76(3): 203-221.

19. Condra J. Resistance to HIV protease inhibitors. Haemophilia 1998; 4(4): 610-615.

20. Gilden D, Falkenberg J, Torres G. Protease inhibitors: resistance, resistance, resistance. GMHC Treat Issues 1997 ; 11(2): 5-10.

21. Gathe, J., Podzamczer, D., Johnson, M. Once-daily vs. twice-daily lopinavir/ritonavir in antiretroviral-naive patients: 48 week results. 11th Conference on Retroviruses and Opportunistic Infections. 2004: 570. 